\title{
DIFERENCIAS ENTRE EL CARÁCTER INSTITUCIONAL Y EL GRADO ESCOLAR EN EL DESEMPEÑO LECTOR EN NIÑOS CON DIAGNÓSTICO DE TDAH*
}

\author{
DIFFERENCES BETWEEN SCHOOLING AND GRADE LEVEL READING PERFORMANCE \\ IN CHILDREN WITH ADHD DIAGNOSTIC
}

\author{
MARÍA ALEXANDRA CARDONA TANGARIFE** \\ UNIVERSIDAD DE MANIZALES, MANIZALES - COLOMBIA \\ HÉCTOR HANEY AGUIRRE-LOAIZA*** \\ CORPORACIÓN EMPRESARIAL UNIVERSITARIA ALEXANDER VON HUMBOLDT, ARMENIA - COLOMBIA \\ UNIVERSIDAD DEL QUINDÍO, ARMENIA - COLOMBIA \\ DIANA MARCELA MONTOYA LONDOÑO***** \\ UNIVERSIDAD DE MANIZALES, MANIZALES - COLOMBIA \\ UNIVERSIDAD DE CALDAS, MANIZALES - COLOMBIA
}

FECHA RECEPCIÓN: 08/08/2014 • FECHA ACEPTACIÓN: 02/10/2014

Para citar este artículo: Cardona, M., Aguirre-Loaiza, H., \& Montoya, D. (2014). Diferencias entre la escolaridad y grado escolar en el desempeño lector en niños con TDAH. Psychologia: avances de la disciplina, 8(2), 13-21.

\begin{abstract}
Resumen
Con los propósitos de identificar y analizar las diferencias entre las características lectoras (precisión, comprensión y velocidad) en función al grado escolar y carácter de la institución, en una muestra intencional de veintitrés niños diagnosticados con TDAH, de ambos géneros (Femenino, $n=9,39.1 \%$; Masculino, $n=14,60.9 \%)$ entre 8 y 11 años $(M=9.13 . D E=1.10)$ de tercer, cuarto y quinto grado escolar de primaria básica, de instituciones oficiales y privadas. Se diseñó un estudio no experimental de corte transversal, y se aplican las tareas de la batería de Evaluación Neuropsicológica Infantil (ENI). Los resultados solamente establecieron diferencias en la comprensión lectora de oraciones con puntajes favorables en niños pertenecientes a colegios privados y de quinto grado. Se concluye que variables escolares permiten diferenciar el desempeño de la comprensión lectora de oraciones en niños diagnosticados con TDAH. Se discuten los resultados y se alientan a nuevos trabajos sobre la misma línea.

Palabras clave: desarrollo infantil, evaluación neuropsicológica, habilidades lectoras, TDAH.
\end{abstract}

\footnotetext{
* Artículo derivado del macroproyecto de investigación denominado: "Caracterización neuropsicopedagogica de niños y niñas con TDAH que asisten a programas de atención en la ciudad de Manizales”, Programa de psicología Universidad de Manizales

** Psicóloga. Universidad de Manizales. Grupo de Investigación de Psicología del Desarrollo. Email: alexacardonatangarife@gmail.com

*** Psicólogo. Licenciado en Educación Física y Deportes. Docente Corporación Empresarial Universitaria Alexander von Humboldt. Universidad del Quindío. Email: haney.aguirre@gmail.com

**** Psicóloga. Mg. en Neuropsicología. Mg. en Educación. Docente programa de Psicología, Universidad de Manizales- Docente Departamento de Estudios Educativos - Universidad de Caldas. email: diana.montoya@ucaldas.edu.co
} 


\begin{abstract}
With the purpose of identifying and analyzing the differences between reading specifications (accuracy, comprehension and speed) according to grade level and nature of institution, either public or private, in a purposive sample of twenty-three children diagnosed with ADHD, from both genders (women, $n=939.1 \%$, male, $n=14,60.9 \%$ ) between 8 and 11 years $(M=9.13, S D=1.10)$ in third, fourth and fifth grade of elementary school, public and private elementary schools. A non-experimental cross-sectional study was designed, and the scale reading Child Neuropsychological Assessment Battery (ENI) is applied. The results established only differences in reading comprehension of sentences with favorable scores in children from private schools and fifth grade. It is concluded that school variables differentiate the performance of reading comprehension of sentences in children diagnosed with ADHD. The results are discussed and encourage further work on the same line. Keywords: Child Development, neuropsychological assessment, reading skills, ADHD.
\end{abstract}

\section{Introducción}

La lectura es un instrumento real para la inclusión social, un factor básico para el desarrollo social, cultural y económico; así lo plantea el informe final de la XIII Cumbre Iberoamericana de Jefes de Estado y Gobierno de Santa Cruz de la Sierra (2003). Es una vía que asegura la libre circulación y apropiación social del conocimiento, una herramienta intelectual indispensable en la construcción de la propia identidad (Organización de Estados Iberoamericanos - OEI, 2004).

La lectura es un proceso que exige una serie de habilidades cognitivas como la atención (Rosselli, Matute, \& Ardila, 2006). Dicha capacidad no es adecuada en las personas diagnosticadas con el Trastorno por Déficit de Atención/Hiperactividad (TDAH), dicho trastorno se identifica como un alteración del comportamiento crónico, y es caracterizado por el inadecuado nivel de atención, impulsividad e hiperactividad (American Psychological Association-APA, 2002; Pascual- Castroviejo, 2004; Zuliani, Uribe, Cardona, \& Cornejo, 2008; Zuluaga, \& Vasco, 2009).

Según las estadísticas, el TDAH es el trastorno más común en la niñez, con cifras de prevalencia que se establecen entre el 3 y $5 \%$ de la población escolar según los registros de la APA (2002). En algunos estudios, la prevalencia aumenta, con datos que oscilan entre el $5 \%$ y $10 \%$ (Scahill, \& Schwab-Stone, 2000) y en cifras colombianas se informan que es del 5.7 \% (Vélez, Toledo, González, \& Ibáñez, 2008); y entre el 13 \% y $22 \%$ (Cornejo et al., 2005).

Así mismo, se estima que entre el 25 y el $40 \%$ de las personas con TDAH también cumplen con los criterios diagnósticos de un trastorno específico del aprendizaje (Willcutt, \& Pennington, 2000); inclusive se afirma que alrededor del $95 \%$ de los niños hiperactivos presentan problemas de aprendizaje (Ardila, Rosselli, \&
Matute, 2005). De modo que la relación entre TDAH y los problemas de aprendizaje ha suscitado particular interés en los investigadores, lo cual es evidenciado en un amplio abordaje investigativo de los diferentes trastornos; por ejemplo: en la escritura (García, Rodríguez, Pacheco, \& Díez, 2009; Miranda, Baixauli, \& Colomer, 2013; Miranda, Soriano, \& García, 2009; Reid, \& Lienemann, 2006), en el cálculo (González, Rodríguez, Fernández, Cabeza, \& Álvarez, 2014; Pérez, Miranda, Meliá, \& Marco, 2009) y en la lectura (Miranda, Jarque, \& Tárraga 2006; Olivera et al., 2013).

A pesar de la asociación evidente, entre TDAH y variables de problemas de aprendizaje, para algunos autores (Miranda-Casas, Fernández, Robledo, \& García-Castellar, 2010), el desarrollo de la investigación en niños con TDAH y habilidades lectoras ha tenido pocos avances, respecto a otros estudios que han tenido más despliegue, como por ejemplo, lo serían estudios realizados en las áreas de cálculo y acceso léxico. Entre los trabajos revisados, se ha podido establecer que el desempeño lector en niños diagnosticados con TDAH ha sido inferior frente a muestras controles (Da Silva, Orlandi, \& Capellini, 2011; Fernández, Miranda, García, \& Colomer, 2011). Similarmente, se han observado resultados análogos en poblaciones de adolescentes (Ghelani, Sidhu, Umesh, \& Tannock, 2004). Las anteriores conclusiones, específicamente, han sido valoradas a partir de la característica lectora de la comprensión; la cual se define como una habilidad compleja en la cual intervienen distintos procesos cognitivos, como la percepción, memoria de trabajo y la resolución de inferencias (Canet-Juric, Urquijo, Richard's, \& Burin, 2009).

Con respecto a la característica lectora de velocidad, entendida como el número de palabras leídas en determinado tiempo (Ardila et al., 2005); se ha evidenciado que en tareas de lectura de palabras y pseudopalabras los niños diagnosticados con TDAH 
tienen mayores dificultades que los niños de grupos de controles (Carro, 2013), lo cual parece estar explicado por la incapacidad de control ejecutivo en las actividades que impliquen tiempo y control voluntario (Bará, Vicuña, Pineda, \& Henao, 2003; Barkley 1997; Capdevila-Brophy et al., 2005; Navarro, \& García-Villamisar, 2011).

En cuanto a la característica de precisión lectora, puede señalarse que esta se entiende como el reconocimiento exacto de las palabras escritas (Ardila et al., 2005), son limitados los estudios encontrados en población diagnosticada con TDAH. Los escasos intentos han determinado diseños comparativos entre grupos con y sin TDAH, confirmándose hipótesis que favorecen significativamente la lectura de palabras en el grupo control, es decir, niños que no son diagnosticados con TDAH (Carro, 2013).

Escasos estudios han vinculado de modo holístico características de precisión, comprensión y velocidad lectora en relación a variables escolares. Por ejemplo, se ha encontrado que aquellos niños que asisten a colegios privados presentaron un mejor desempeño lector que los niños de colegios públicos (Roselli, Matute, \& Ardila, 2006); tales desarrollos han sido de relativo avance, y no se conocen trabajos focalizados en poblaciones infantiles con TDAH. No existe suficiente claridad y desde luego, sin evidencia empírica de las características lectoras de los niños con TDAH respecto a las variables escolares como el grado de escolaridad y el carácter de la institución (pública o privada). Por lo tanto, es pertinente plantear como hipótesis alternativa para el presente trabajo, la existencia de diferencias en el comportamiento lector (precisión, comprensión y velocidad) en función al grado y el carácter de la institución en niños con diagnóstico de TDAH. Este trabajo implicaría impactos sobre el conocimiento de variables socioambientales que pueden ser decisivas en la toma de decisiones de programas escolares, intervenciones psicopedagógicas y políticas gubernamentales para efectos del desempeño lector en poblaciones infantiles con las características previamente mencionadas.

En consecuencia, el presente estudio, bajo la pretensión de contribuir en el cuerpo de conocimiento específico del área, se planteó como objetivo identificar y analizar las diferencias entre las características lectoras (precisión, comprensión y velocidad) en función a variables escolares del grado escolar y el carácter institucional en niños diagnosticados con TDAH.

\section{Método}

\section{Diseño}

Para cumplir los objetivos trazados se efectuó un estudio no experimental de corte transversal (Hernández-Sampieri, Fernández, \& Baptista, 2014), debido a que no fueron manipuladas ninguna de las variables independientes.

\section{Participantes}

A partir de un diseño muestral por conveniencia o intencional, en el estudio se contó con una muestra de 23 niños de ambos géneros (Varones $n=9,39.1 \%$ y Mujeres $n=14,69.9 \%$ ) de la ciudad de Manizales, Colombia. Con edades comprendidas entre 8 y 11 años $(M=9.13 . D E=$ 1.10). La distribución de los niños por edades correspondió a: 8 años $(n=9,39.1 \%), 9$ años $(n=5,21.7 \%), 10$ años $(n=6,26.1 \%)$, y los de 11 años $(n=3,13.0 \%)$. De acuerdo con el subtipo de TDAH, el subgrupo de inatento $(n=10,43.5 \%)$ y el grupo de combinado $(n=$ 13, $56.5 \%)$. De instituciones de carácter oficial $(n=11$, $47.8 \%$ ) y privado ( $n=12,52.2 \%)$; al igual que los grados escolares, se encontró de tercer grado $(n=12,52.2 \%)$, de cuarto grado $(n=3,13.0 \%)$ y de quinto grado $(n=8$, $34.8 \%$ ). Los criterios de inclusión para la muestra correspondieron a la ausencia de comorbilidades y Coeficiente Intelectual (CI) mayor a 80.

\section{Técnicas e instrumentos}

Para evaluar las características lectoras se aplicaron las tareas de lectura de la Batería de la Evaluación Neuropsicológica Infantil (ENI) de Matute, Roselli, Ardila y Ostrosky-Solís (2007), cuyo diseño fue establecido para niños con edades entre 5 y 16 años. El grupo normativo corresponde a una muestra $(n=788)$ niños de las ciudades de Manizales (Colombia) y de Guadalajara (México). La confiabilidad y validez entre calificadores de las secciones del ENI oscilan entre un coeficiente de .85 y .98 .

Para el diagnóstico de TDAH se empleó el cuestionario breve de Checklist, utilizado en contextos colombianos (Pineda et al., 1999). La consistencia interna de la prueba arrojó un coeficiente de alpha de Cronbach para los diferentes ítems entre .85 y .92, aplicados a una muestra de niños y niñas de la ciudad de Manizales (Colombia), con edades entre 4 y 17 años. Así mismo, la 
entrevista psiquiátrica semiestructurada (MINI KID) para niños y adolescentes (Sheehan, Lecrubier, \& Colón-Soto, 2000; Sheehan, Shytle, Milo, Lecrubier, \& Hergueta, 2005). Finalmente se aplicó el Test de Inteligencia para niños de Wechsler (WISC-III) abreviado en la forma C6 $\mathrm{X} 2$, con las subpruebas de vocabulario y diseño con cubos (Sattler, 2003), cuyo puntaje permitió determinar el criterio de inclusión referido a la medida de CI.

\section{Procedimiento y consideraciones éticas}

Se contactó con los directivos y equipo de apoyo interdisciplinario de las instituciones educativas. Una vez brindada la autorización para la participación en la institución se procedió a aplicar instrumentos de tamizaje. La búsqueda se realizó con aquellos niños y niñas cuya puntuación T fue mayor a 60 en el cuestionario breve de Checklist por considerarlos casos sospechosos de TDAH.

A los sujetos escogidos mediante esta estrategia, se les administró una prueba WISC III abreviado, según la forma C6 x2 (Sattler, 2003) para estimar su capacidad intelectual. Quienes obtuvieron un CI igual o superior a 85 fueron evaluados en una entrevista psiquiátrica estructurada a través de la (MINI KID) para determinar el cumplimiento de criterios clínicos para TDAH.

Acorde a la información brindada a los padres de los niños participantes y obtención de los respectivos consentimientos informados, y siguiendo los lineamentos del Código Ético y Deontológico del Colegio Colombiano del Psicólogo (2012), se procedió a programar las jornadas requeridas para el proceso de evaluación y diagnóstico. Este proceso dispuso de tres sesiones, cada una, aproximadamente entre 40 y 45 minutos de duración, discriminadas así: entrevista psiquiátrica semiestructurada, ejecutada por un psiquiatra entrenado en el uso de la MINI KID y Cheklist, en la segunda sesión administración del tamizaje de capacidad intelectual (medida que fue utilizada solo como criterio de inclusión) y finalmente, se aplicaron las subpruebas de lectura de la ENI.

\section{Análisis estadístico de los datos}

Los datos fueron procesados en dos momentos: en una primera instancia se efectuó una depuración y filtración a través de una matriz de Excel de Microsoft Windows. Posteriormente se trasladaron al paquete estadístico de SPSS v.17.0. Para establecer los cálculos descriptivos se emplearon las medidas de tendencia central (promedio) y de dispersión (la desviación estándar). La estadística inferencial fue calculada a partir de las pruebas no perimétricas de U Mann-Withney para dos muestras independientes, y para más de dos muestras a través Kruskall-Wallis, debido a que el supuesto de homogeneidad no fue aprobado según la prueba de Normalidad de Shapiro-Wilk $(n=23, p<0.05)$.

\section{Resultados}

Los puntajes promedios generales de las características específicas de cada uno de los componentes de las variables de precisión, comprensión y velocidad son descritas en la tabla 1. Los datos indican que las medias generales más sobresalientes correspondieron a la precisión lectura de oraciones $(M=9.9, D E=2.8)$, seguido de la lectura de sílabas $(M=9.7, D E=2.7)$. En tanto, los puntajes más bajos se observaron específicamente en el componente de precisión en el error de lectura en voz alta $(M=6.7, D E=3.3)$, y en el componente de velocidad de lectura en voz alta $(M=6.6, D E=3.6)$.

Tabla 1. Promedios generales del puntaje natural y escalar de las características lectoras

\begin{tabular}{lllll}
\hline \hline $\begin{array}{l}\text { Variables } \\
\text { de lectura }\end{array}$ & \multicolumn{2}{c}{$\begin{array}{c}\text { Puntaje } \\
\text { natural }\end{array}$} & \multicolumn{2}{c}{$\begin{array}{c}\text { Puntaje } \\
\text { escalar }\end{array}$} \\
\hline \hline Precisión & $\mathrm{M}$ & $\mathrm{DE}$ & $\mathrm{M}$ & $\mathrm{DE}$ \\
\cline { 2 - 5 }$\quad$ Sílabas & 7.5 & 0.7 & 9.7 & 2.7 \\
$\quad$ Palabras & 10.7 & 0.5 & 9.6 & 1.6 \\
$\quad$ No palabras & 6.9 & 0.9 & 9.5 & 2.2 \\
$\quad$ Oración & 9.0 & 1.1 & 9.9 & 2.8 \\
$\quad$ Error en voz alta & 6.0 & 3.9 & 6.7 & 3.3 \\
Comprensión & & & & \\
$\quad$ Oraciones & 7.1 & 1.5 & 8.0 & 2.8 \\
$\quad$ Texto en voz alta & 4.1 & 2.1 & 9.6 & 4.2 \\
$\quad$ Texto silencioso & 3.5 & 1.5 & 9.5 & 2.6 \\
Velocidad & & & & \\
$\quad$ Texto en voz alta & 59.9 & 27.7 & 6.6 & 3.6 \\
$\quad$ Texto silencioso & 70.8 & 55.2 & 7.4 & 4.4 \\
\hline \hline
\end{tabular}

Nota: Una mejor puntuación en el promedio indica mejor desempeño lector 


\section{Carácter de la institución educativa y variables de lectura}

Los resultados del comportamiento lector en función al carácter de la institución se describen en la tabla 2. Los hallazgos establecen diferencias significativas en la variable de comprensión lectora en oraciones $(U=20.5, p=.005)$, siendo con mayor puntaje en las instituciones privadas $(M=$ 9.4, $D E=2.9)$ en comparación a las instituciones estatales $(M=6.4, D E=1.7)$. A pesar de que las demás características no arrojaron diferencias estadísticamente significativas, la tendencia de puntajes beneficia a las instituciones privadas, solo la media de la precisión lectora de sílabas fue superior en los sujetos que provienen de una institución oficial. Llama la atención el puntaje igualado entre ambas instituciones, en los puntajes de la precisión del error en lectura de voz alta $(M=6.7)$, y la comprensión en texto silencioso $(M=9.5)$.

Tabla 2. Promedios de los puntajes escalares en las variables de lectura según el carácter de la institución educativa

\begin{tabular}{|c|c|c|c|c|c|c|}
\hline \multirow{3}{*}{$\begin{array}{l}\text { Variables de } \\
\text { lectura }\end{array}$} & \multicolumn{4}{|c|}{$\begin{array}{c}\text { Carácter de la } \\
\text { institución educativa }\end{array}$} & \multirow{3}{*}{$\mathrm{U}$} & \multirow{3}{*}{$\mathrm{p}$} \\
\hline & \multicolumn{2}{|c|}{$\begin{array}{c}\text { Oficial } \\
(n=11)\end{array}$} & \multicolumn{2}{|c|}{$\begin{array}{l}\text { Privado } \\
(n=12)\end{array}$} & & \\
\hline & M & $\mathrm{DE}$ & M & $\mathrm{DE}$ & & \\
\hline \multicolumn{7}{|l|}{ Precisión } \\
\hline Sílabas & 10.8 & 0.4 & 8.7 & 3.4 & 42.0 & .080 \\
\hline Palabras & 9.2 & 2.4 & 10.0 & 0.0 & 60.0 & .296 \\
\hline No Palabras & 9.2 & 2.3 & 9.8 & 2.0 & 57.0 & .564 \\
\hline Oración & 9.3 & 3.4 & 10.4 & 2.1 & 62.0 & .792 \\
\hline $\begin{array}{l}\text { Error en } \\
\text { voz alta }\end{array}$ & 6.7 & 3.3 & 6.7 & 3.4 & 65.0 & .950 \\
\hline \multicolumn{7}{|l|}{ Comprensión } \\
\hline Oraciones & 6.4 & 1.7 & 9.4 & 2.9 & 20.5 & $.005 *$ \\
\hline $\begin{array}{l}\text { Texto en } \\
\text { voz alta }\end{array}$ & 9.3 & 4.2 & 9.9 & 4.4 & 59 & .665 \\
\hline $\begin{array}{l}\text { Texto } \\
\text { silencioso }\end{array}$ & 9.5 & 2.2 & 9.5 & 3 & 65.5 & .975 \\
\hline \multicolumn{7}{|l|}{ Velocidad } \\
\hline $\begin{array}{l}\text { Texto en } \\
\text { voz alta }\end{array}$ & 6.3 & 3.7 & 6.8 & 3.7 & 63.5 & .877 \\
\hline $\begin{array}{l}\text { Texto } \\
\text { silencioso }\end{array}$ & 7.2 & 4.7 & 7.5 & 4.2 & 57.5 & .594 \\
\hline
\end{tabular}

Nota: Una mejor puntuación en el promedio indica mejor desempeño lector. En negrilla los puntajes superiores. $* p<0.01$

\section{Grado escolar y variables de lectura}

La variable grado escolar describe el análisis estadístico de las submuestras de los grados tercero, cuarto y quinto de básica primaria en la tabla 3. Se observa que el puntaje promedio de la precisión lectora en sílabas, la comprensión lectora de texto en voz alta y la velocidad lectora, tanto en voz alta, como en texto silencioso, fue superior en los estudiantes de tercer grado comparados con los grupos de cuarto y quinto grado. Por otro lado, la comprensión lectora de oraciones corresponde a la variable donde se establecieron diferencias significativas entre dichos grados de escolaridad, $X_{(2, N=23)}^{2}=12.5$, $p=.002$, arrojando promedios superiores a favor del grado quinto en contraste con al grado tercero y cuarto.

Tabla 3. Promedios de los puntajes escalares en las variables de lectura según el grado escolar

\begin{tabular}{|c|c|c|c|c|c|c|c|c|}
\hline \multirow{3}{*}{$\begin{array}{l}\text { Variables de } \\
\text { lectura }\end{array}$} & \multicolumn{6}{|c|}{ Grado escolar } & \multirow{3}{*}{$\begin{array}{l}X^{2} \\
(2)\end{array}$} & \multirow{3}{*}{$\mathrm{p}$} \\
\hline & \multicolumn{2}{|c|}{$\begin{array}{l}\text { Tercero } \\
(n=11)\end{array}$} & \multicolumn{2}{|c|}{$\begin{array}{l}\text { Cuarto } \\
(n=3)\end{array}$} & \multicolumn{2}{|c|}{$\begin{array}{l}\text { Quinto } \\
(\mathrm{n}=8)\end{array}$} & & \\
\hline & $\mathrm{M}$ & $\mathrm{DE}$ & M & $\mathrm{DE}$ & $\mathrm{M}$ & $\mathrm{DE}$ & & \\
\hline \multicolumn{9}{|l|}{ Precisión } \\
\hline Sílabas & 10.5 & 0.5 & 8.3 & 4.6 & 9 & 3.7 & 0.11 & .946 \\
\hline Palabras & 9.3 & 2.3 & 10.0 & 0.0 & 10 & 0.0 & 0.91 & .632 \\
\hline $\begin{array}{l}\text { No } \\
\text { Palabras }\end{array}$ & 9.4 & 2.6 & 11.3 & 1.1 & 9.1 & 1.9 & 2.79 & .247 \\
\hline Oración & 9.6 & 3.1 & 11.6 & 0.5 & 9.6 & 2.8 & 1.01 & .602 \\
\hline $\begin{array}{l}\text { Error en } \\
\text { voz alta }\end{array}$ & 7.0 & 3.4 & 6.3 & 3.0 & 6.4 & 3.6 & 0.88 & .644 \\
\hline \multicolumn{9}{|l|}{ Comprensión } \\
\hline Oraciones & 7.3 & 1.8 & 4.0 & 2.0 & 10.5 & 2.0 & 12.5 & $.002 *$ \\
\hline $\begin{array}{l}\text { Texto en } \\
\text { voz alta }\end{array}$ & 11.2 & 3.3 & 8.0 & 2.0 & 7.8 & 5.3 & 3.11 & .211 \\
\hline $\begin{array}{l}\text { Texto } \\
\text { silencioso }\end{array}$ & 9.6 & 2.9 & 10.0 & 2.6 & 9.1 & 2.4 & 0.24 & .886 \\
\hline \multicolumn{9}{|l|}{ Velocidad } \\
\hline $\begin{array}{l}\text { Texto en } \\
\text { voz alta }\end{array}$ & 6.9 & 3.9 & 7 & 4.3 & 6 & 3.4 & 0.70 & 0.70 \\
\hline $\begin{array}{l}\text { Texto } \\
\text { silencioso }\end{array}$ & 8.4 & 5.6 & 6.3 & 1.5 & 6.3 & 2.7 & 0.97 & 0.61 \\
\hline
\end{tabular}

Nota: Una mejor puntuación en el promedio indica mejor desempeño lector. En negrilla los puntajes superiores.

$* p<0.01$ 


\section{Discusión}

El objetivo que se pretendió en este estudio, correspondió a identificar y analizar las diferencias entre la precisión, comprensión y velocidad lectora en niños diagnosticados con TDAH, en función al grado escolar y el carácter de la institución educativa. Del mismo modo, guiados por la hipótesis de trabajo, se asumía la existencia de diferencias significativas en el comportamiento lector de dichas características (precisión, comprensión y velocidad lectora), ante lo cual, los resultados confirman parcialmente la hipótesis alternativa, al solo comprobarse diferencias significativas en la comprensión de oraciones en las variables estudiadas de grado escolar y la institución educativa. No fueron observadas diferencias en las otras características lectoras.

Es decir, los resultados del presente trabajo observaron promedios estadísticamente significativos con mejor desempeño de comprensión lectora de oraciones en niños de grado quinto comparados frente a los homólogos de grado cuarto y tercero, iguales resultados fueron establecidos en la variable del carácter institucional, así, niños que asisten a instituciones educativas cuyo carácter es privado, arrojaron valores promedios superiores en comparación a los niños que asisten a instituciones educativas de carácter oficial.

Frente a la diferencia del grado escolar, los datos arrojados en el presente estudio coinciden con el trabajo de Bolaños-García y Gómez-Betancurt (2009), donde se han descrito promedios de la comprensión lectora de oraciones en niños escolarizados con trastornos de aprendizaje, dicho desempeño fue superior para los grados quintos, sobre los grupos de cuarto y tercero. Aunque en dicho trabajo no se establecieron diferencias significativas en las submuestras (grados escolares), sí se pudo establecer la dirección de los puntajes promedios, identificándose finalmente que aquellos sujetos de grado quinto estuvieron por encima de cuarto y tercero, y por lo tanto, mejor desempeño de la característica específica de la comprensión lectora en oraciones. El puntaje favorable para los niños de grado quinto, dan un respaldo en la perspectiva que adquiere el desarrollo escolar en los niños con TDAH, además del desenvolvimiento neuropsicológico principalmente en dimensiones lectoras las cuales se destacan con mayor énfasis durante estos periodos escolares (Roselli et al., 2006; 2010).
Un aspecto que llama la atención en el componente de comprensión lectora de oraciones, es que puntaje obtenido por los niños del grado cuarto obtuvo la cifra más baja en, inclusive inferior a la de los sujetos de tercer grado. Este bajo desempeño puede ser considerado como una característica del bajo rendimiento escolar y por ende, que se enmarca en el diagnóstico del TDAH, es decir, parece ser que dicho grado escolar, se comprende como un periodo donde habitualmente se consolida la lectoescritura como base del aprendizaje escolar, y en algunos casos no se ha contado con la intervención adecuada (Montoya-Londoño, Varela-Cifuentes, \& Dussán-Lubert, 2011).

Adicionalmente, otro elemento que debe tenerse en cuenta, y que plausiblemente permite explicar el anterior aspecto, es que algunos niños cuyo diagnóstico ha sido tardío (después de los 10 y/u 11 años) presentan mayor porcentaje de un pobre rendimiento escolar que el resto de grupos diagnosticados oportunamente, por consiguiente, se observa una afectación del desempeño escolar general, y desde luego, desfavorece en las características lectoras (García-Pérez, Expósito-Torrejón, Martínez-Granero, Quintanar-Rioja, \& Bonet-Serra, 2005), de modo que los comportamientos del desempeño lector pueden variar según la edad del diagnóstico, puesto que la intervención oportuna de los profesionales facilitará procesos escolares que contribuyan al niño y su entorno. Posteriormente, es importante advertir que ante los datos presentados en la variable del grado de escolar, deben asumirse con discreción puesto que las submuestras no están igualadas, y así, algunos de los promedios y varianzas del desempeño lector pueden beneficiar a un grado sobre otro.

Con respecto a la diferencia del carácter de la institución educativa, se pueden entender aspectos sociocontextuales que estiman cierta favorabilidad de los colegios de carácter privado frente a aquellos de carácter oficial o público, lo cual puede ser entendido por factores como el número de estudiantes, características de infraestructura física, elementos didácticos y de procesos de acompañamiento psicopedagógico. En términos generales, se ha considerado que el carácter de la institución es uno de los criterios que puede tener algún tipo de efecto en el comportamiento neuropsicológico del desarrollo infantil (Roselli et al., 2006) lo cual es evidenciado específicamente en las habilidades lectoras tal y como se corrobora en los presentes resultados. 
Algunos trabajos descriptivos (De los Reyes, Lewis, \& Peña, 2008), previamente han efectuado aproximaciones, concluyendo que el $3.3 \%$ de escolares de colegios privados en una población infantil han presentado dificultades de lectura; no obstante, estos resultados se establecen con cautela dado los alcances metodológicos del estudio referenciado. En consecuencia, el carácter de la institución educativa debe ser tenida en cuenta en futuros estudios para corroborar tales supuestos, al igual que tener presente posibles variables ambientales (p. ej. formación y nivel de escolaridad del docente, así como del padre y/o madre de familia, número de estudiantes compañeros, programa curricular, etc.) y la relación del TDAH con el rendimiento escolar, dado que previos informes han sugerido afectaciones sobre el rendimiento escolar en niños de una institución privada (Moreno \& Martínez, 2012).

En síntesis, frente al componente de comprensión lectora es importante destacar, que un buen nivel de comprensión lectora siguiendo el trabado de Canet-Juric et al. (2009) implica ser eficiente en el uso de habilidades lingüísticas (monitoreo, inferencias, vocabulario) y disponer de habilidades de procesamiento (memoria de trabajo). Por lo tanto, variables como el nivel de grado escolar y el carácter de la institución educativa señalan diferencias del rendimiento comprensivo en niños con TDAH.

Frente al desempeño de precisión y velocidad lectora, los datos no efectuaron diferencias significativas, lo que puede entenderse como características de amplia complejidad y que son de comportamiento uniforme en los niños con TDAH; en este contexto se deduce que las instituciones educativas cuyo carácter sea oficial o privado, así como el grado de escolaridad no evidencian discrepancias. No obstante, es meritorio destacar que las variables lectoras son desfavorables en muestras con diagnóstico de TDAH reportando valores significativamente inferiores en comparación a muestras controles (Da Silva et al., 2011; Fernández et al., 2011; Ghelani et al., 2004; Olivera et al., 2013).

No está de más, advertir algunas de las limitantes del presente estudio, y a su vez, asumir una serie de recomendaciones para futuros trabajos, por ejemplo, a nivel metodológico, considerar muestras de controles y emparejar los sujetos en función a variables independientes (p. ej., género, edad, grado escolar, estrato socioeconómico, etc.) con el fin de evitar posibles aspectos de invalidez que sean amenazantes; de la misma manera, evaluar el rendimiento lector por medio de otros instrumentos y revisar la sensibilidad de los mismos en niños diagnosticados con TDAH, dado que el nivel de la dificultad de la tarea asume mayor compromiso y complejidad por parte del desempeño lector, y más aún, cuando se reconoce que las dificultades lectoras tienen relación con el déficit del funcionamiento ejecutivo (Locascio, Mahone, Eason, \& Cutting, 2010), igualmente contemplar rangos inferiores de los grados escolares, así como se había mencionado previamente, estudiar los niveles de formación del docente y de los padres de los niños participantes.

Finalmente, este estudio sostiene que la comprensión lectora de oraciones en función a variables como el grado escolar y el carácter del colegio, evidencia diferencias en niños de tercero, cuarto y quinto grado de primaria, de colegios privados y estatales. Lo cual implica, principalmente, que los profesionales de la educación y de la salud mental, pueden asumir determinados factores escolares, como elementos importantes en las habilidades lectoras y por ende, el desempeño escolar del niño, principalmente en lo que concierne a la comprensión lectora.

Así entendido, el fortalecimiento de programas de acompañamiento psicopedagógico y estrategias políticas que impacten en el ámbito educativo en niños diagnosticados con TDAH hace parte de uno de los pilares que debe asumirse en instituciones de carácter público o estatal. En esta lógica, son necesarios otros trabajos en estas líneas que permitan nutrir avances en la construcción de conocimiento sobre los factores asociados y las diferencias en las características lectoras de los niños diagnosticados con TDAH.

\section{Referencias}

Ardila, A., Rosselli, M., \& Matute, E. (2005). Neuropsicología de los trastornos del aprendizaje. México: Manual Moderno.

Asociación Americana de Psiquiatría. (2002). Manual diagnóstico y estadístico de los trastornos mentales. (DSMIV-TR). Bogotá: Masson.

Bará, S., Vicuña, P., Pineda, D., \& Henao, G. (2003). Perfiles neuropsicológicos y conductuales de niños con trastorno por déficit de atención/ 
hiperactividad de Cali, Colombia. Revista Neurología, 37(7), 608-615.

Barkley, R. A. (1997). Behavioral inhibition, sustained attention, and executive functions: constructing a unifying theory of ADHD. Psychological Bulletin, $121(1), 65$.

Bolaños, R., \& Gómez, L. (2009). Características lectoras de niños con trastorno del aprendizaje de la lectura. Acta Colombiana de Psicología, 12 (2), 37-45.

Carro, N. M. (2013). Análisis de los procesos lectores en un grupo de niños con trastorno por déficit de atención con hiperactividad (TDAH). Recuperada de repositorio Universidade da Coruña. http://ruc.udc.es/dspace/handle/2183/11852

Canet-Juric, L., Urquijo, S., Richard's, M.M., \& Burin, D. (2009). Predictores cognitivos de niveles de comprensión lectora mediante análisis discriminante. International Journal of Psychological Research, 2(2), 99-111.

Capdevila-Brophy, C., Artigas-Pallarés, J., RamírezMallafré, A., López-Rosendo, M., Real, J., \&ObiolsLlandrich, J. E. (2005). Fenotipo neuropsicológico del trastorno de déficit atencional/hiperactividad: ¿existen diferencias entre los subtipos? Revista de Neurología, 40(Supl 1), S17-S23.

Cornejo, J., Osío, O., Sánchez, Y., Carrizosa, J. Sánchez, G. Grisales, H. Castillo-Parra, H., \& Holguín, J. (2005). Prevalencia del trastorno por déficit de atenciónhiperactividad en niños y adolescentes colombianos. Revista de Neurología, 40 (12), 716 -722.

Colegio Colombiano de Psicólogos. (2012). Deontología y bioética del ejercicio de la psicología en Colombia (3. ${ }^{a}$ ed.). Bogotá: Colegio Colombiano de Psicólogos.

Cumbre Iberoamericana de Jefes de Estado y Gobierno (2003). Cumbre Iberoamericana Santa Cruz de la Sierra. Recuperado de: http://www.redipd.org/actividades/ common/xiii_cumbre/Declaracion_Santa_Cruz_ de_la_Sierra_Bolivia.pdf

Da Silva, C., Orlandi, V., \& Capellini, S. (2011). Performance cognitive-linguistic and reading of students with attention deficit and hyperactivity disorder. Journal of Human Growth and Development, 21 (3), 849-858.

De los Reyes, C., Lewis, S., \& Peña, M. (2008). Estudio de prevalencia de dificultades de lectura en niños escolarizados de 7 años de Barranquilla (Colombia). Psicología desde el Caribe, 22, 37-49.

Fernández, M. I., Miranda, A., García, R., \& Colomer, C. (2011). Diferencias entre sujetos con TDAH con y sin dificultades en comprensión lectora: los procesos de la comprensión lectora. International Journal of Developmental and Educational Psychology, 1 (3), 277-286.

García-Pérez, A., Expósito-Torrejón, J., MartínezGranero, M., Quintanar-Rioja, A., \& Bonet-Serra, B. (2005). Semiología clínica del trastorno por déficit de atención con hiperactividad en función de la edad y eficacia de los tratamientos en las distintas edades. Revista de Neurología, 41 (9), 517-524.

García, J. N., Rodríguez, C., Pacheco, D., \& Diez, C. (2009). Influencia del esfuerzo cognitivo y variables relacionadas con el TDAH en el proceso y producto de la composición escrita. Un estudio experimental. Estudios de Psicología, 30(1), 31-50.

Ghelani, K., Sidhu, R., Umesh, J., \& Tannock, R. (2004). Reading comprehension and reading related abilities in adolescents with reading disabilities and attention deficit/hyperactivity disorder. Dyslexia, 10, 364-384.

González, P. G., Rodríguez, C., Fernández, M. S., Cabeza, L., \& Álvarez, L. A. (2014). Competencias matemáticas y control ejecutivo en estudiantes con trastorno por déficit de atención con hiperactividad y dificultades de aprendizaje de las matemáticas. Revista de Psicodidáctica, 19(1), 125-143.

Hernández-Sampieri, R., Fernández, C., \& Baptista, P. (2014). Metodología de la investigación. (6. ed.) México: McGraw-Hill.

Locascio, G., Mahone, E. M., Eason, S. H., \& Cutting, L. E. (2010). Executive dysfunction among children with reading comprehension deficits. Journal of Learning Disabilities, 43(5), 441-454.

Matute, E., Roselli, M., Ardila, A., \& Ostrosky-Solís, F. (2007). Evaluación Neuropsicológica Infantil. México D. F.: Manual Moderno.

Miranda-Casas, A., Fernández, M. I., Robledo, P., \& García-Castellar, R. (2010). Comprensión de textos de estudiantes con trastorno por déficit de atención/hiperactividad: ¿Qué papel desempeñan las funciones ejecutivas? Revista de Neurología, 50 (supl.3), S135.42 
Miranda, A., García, R., \& Jara, P. (2001). Acceso al léxico y comprensión lectora en los distintos subtipos de niños con trastornos por déficit de atención con hiperactividad. Revista de Neurología Clínica, 2(1), 125-138.

Miranda, A., Jarque, S., \& Tárraga, R. (2006). Interventions in school settings for students with ADHD. Exceptionality, 14(1), 35-52.

Miranda, A., Meliá, A., \& Marco, R. (2009). Habilidades matemáticas y funcionamiento ejecutivo de niños con TDAH. Psicothema, 21, 63-69.

Miranda, A., Soriano, M., \& García, R. (2006). Reading comprehension and written composition problems of children with ADHD: Discussion of research and methodological considerations. Advances in Learning and Behavioral Disabilities, 19, 237-256.

Miranda A., Baixauli I., \& Colomer C. (2013). Narrative writing competence and internal state terms of young adults clinically diagnosed with childhood attention deficit hyperactivity disorder. Research in Developmental Disabilities, 3(6), 1338-1350.

Montoya-Londoño, D., Varela-Cifuentes, V., \& Dussán, C. (2011).Caracterización neuropsicología de una muestra de niños y niñas con TDAH de la ciudad de Manizales. Biosalud, 10 (1), 30-51.

Moreno, J., \& Martínez, N. (2012). Conductas externalizantes, rendimiento académico y atención selectiva en niños con y sin hiperactividad. Psychologia: avances de la disciplina, 4(1), 39-53.

Navarro, M., \& García-Villamisar, D. A. (2011). Funcionamiento ejecutivo en el trastorno de déficit de atención con hiperactividad: una perspectiva ecológica de los perfiles diferenciales entre los tipos combinado e inatento. Revista de Psicopatología y Psicología Clínica, 16(2), 113-124.

Olivera, A., Cardoso, M., Padula, N., Lourencetti, M., Santos, L. C., \& Capellini, S. (2013). Processos de leituraem escolares com Trastorno de Déficit de Atencao/ Hiperatividade. Psicología Argumento, 31 (72), 35-44.

Organización de Estados Iberoamericanos - OEI. (2004). Agenda de políticas públicas de lectura. Bogotá: OEI.

Pascual-Castroviejo, I. (2004). Síndrome de déficit de atención con hiperactividad y capacidad para el deporte. Revista de Neurología, 38 (11), 1001-1005.

Pineda, D. A., Henao, G. C., Puerta, I. C., Mejía, S. E., Gómez, L. F., Miranda, M. L., \& Murrelle,
L. (1999). Uso de un cuestionario breve para el diagnóstico de deficiencia atencional. Revista Neurológica, 28(4), 365-72.

Reid, R., \& Lienemann, T. O. (2006). Self-Regulated Stra- tegy Development for Written Expression in Students with Attention Deficit/Hyperactivity Disorder. Exceptional Children, 73(1), 53-68.

Roselli, M., Matute, E., \& Ardila, A. (2006). Predictores neuropsicológicos de la lectura en español. Revista de Neurología, 42 (4), 2020-210.

Roselli, M., Matute, E., \& Ardila, A. (2010). Neuropsicología del desarrollo infantil. México: Manual Moderno.

Sattler, J. M. (2003). Evaluación infantil: aplicaciones conductuales y clínicas. México: Manual Moderno.

Scahill, L., \& Schwab-Stone, M. (2000). Epidemiology of ADHD in school-age children. Child and Adolescent Psychiatric clinics of North America, 9(3), 541-555.

Sheehan, D., Lecrubier, Y., \& Colón-Soto (2000). MINI KID. Mini International Neuropsychiatric Interview para niños y adolescentes. Recuperado en. www. medicaloutcomes.com.

Sheehan, D., Shytle, D., Milo, K., Lecrubier, Y., \& Hergueta, T. (2005). Mini International Neuropsychiatric Interview. Versión en español. Recuperado en. www.medicaloutcomes.com.

Vélez, A., Toledo, C., González, R., \& Ibáñez, M. (2008). Prevalencia de trastorno por déficit de atención con hiperactividad en estudiantes de escuelas de Bogotá, Colombia. Acta Neurología Colombiana. 24 (1), 6-12.

Willcutt, E., \& Pennington, B. (2000). Psychiatric Comorbidity in Children and Adolescentswith Reading Disability. Journal of Child Psychology and Psychiatry, 41 (8), 1039-1048.

Zuluaga J. B., \& Vasco, C. E. (2009). Evolución en la atención, los estilos cognitivos y el control de la hiperactividad en niños y niñas con diagnóstico de trastorno deficitario de atención con hiperactividad (TDAH). Revista Latinoamericana de Psicología. 41 (3), 481-496.

Zuliani, L., Uribe, M., Cardona, J., \& Cornejo, J. (2008). Características clínicas, neuropsicológicas y sociodemográficas de niños varones con déficit de atención/hiperactividad de tipo inatento en Medellín, Antioquia, Colombia, 2004-2005. Iatreia, 21 (4), 375, 384. 